\title{
Forecasting Household Electricity Consumption Using Time Series Models
}

\author{
Patcharakorn Sokannit and Pasapitch Chujai
}

\begin{abstract}
The present study sought to develop and compare models for forecasting time series data on the household electricity consumption using ARIMA, GARCH and Winters Triple Exponential Smoothing models. Two datasets used in this study were monthly time series data from Provincial Electrical Authority, Punpin district, Suratthani province; specifically, the datasets were concerned with the household electricity consumption fewer than and above 150 units. The selection of the optimal forecasting model was based on the lowest RMSE. The results demonstrated that the GARCH models, namely GARCH $(2,0)$ and GARCH $(1,1)$ respectively, were suitable for the time series data on the household electricity consumption below and above 150 units; the two models could be used to provide only a one-month ahead forecast.
\end{abstract}

Index Terms-ARIMA, GARCH, triple exponential smoothing (Winter), time series data on household electricity consumption, stationary.

\section{INTRODUCTION}

Electricity is inevitably one of the essential factors in life; it is clean energy and has greater ease of use than any other energy. Needs for electricity consumption tend to grow as the economy is flourishing and the technological advancement is growing. In fact, Thailand has 42,000 megawatts of generation capacity; the proportion of fuel consumption for electricity generation of Electricity Generating Authority of Thailand in 2019 consisted of the following elements: 61.20 percent of natural gas, 22.67 of coal/lignite, 14.73 of renewable energy as in hydropower, 0.47 of fuel oil, 0.07 of diesel fuel and 0.86 of any other sources as in Laos, Malaysia and Lamtakong Energy [1]. The proportion of generated electricity consumption of each sector is composed as follows 0.2 percent of NGOS, 0.01 of agriculture, 1.7 of free of charge electricity, 24 of household use, 24 of commercial use, 48 of industrial use and 2.3 of other sectors; the means of electricity consumption in each sector has been increasing [2]. Among those factors, household electricity consumption particularly tends to grow exponentially, as can be seen in Fig. 1, which displays household electricity consumption from 2009 to 2018, Department of Alternative Energy Development and Efficiency, Ministry of Energy.

In 2009 , the electricity consumption was 20,475 million

Manuscript received March 3, 2020; revised February 19, 2021. This work was supported by the Electrical Technology Education Department, Faculty of Industrial Education and Technology, KMUTT.

P. Sokannit is with the Faculty of Education, Industry and Technology, King Mongkut's University of Technology Thonburi 10140, Thailand (email: eyepatkorn@gmail.com).

P. Chujai is with the Electrical Technology Education Department, Faculty of Industrial Education and Technology, King Mongkut's University of Technology Thonburi, Bangkok, Thailand (e-mail: pasapitchchujai@gmail.com). kilowatt-hours; until 2017, it increased up to 32,078 million. It is clear that there has been a continuous growth in the electricity consumption, which is consistent with time (year).

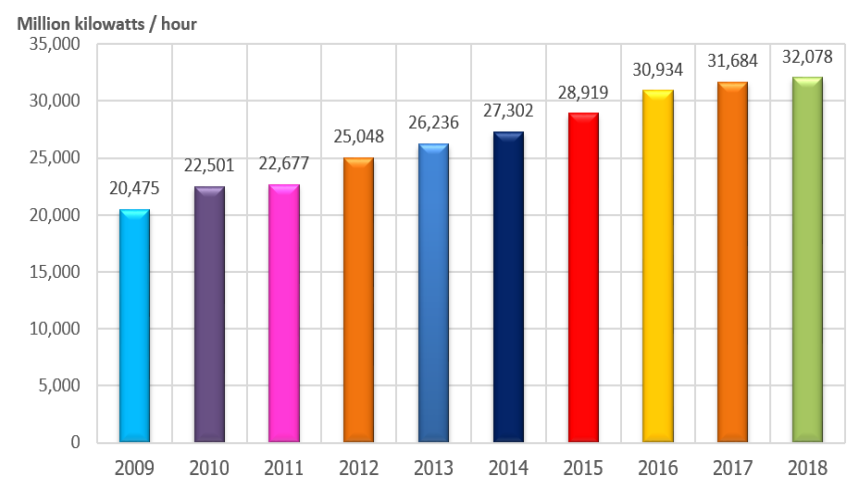

Fig. 1. Household electricity consumption from 2009 to 2018 [3].

The constant rise in electricity consumption indicates the citizens' growing needs or demands. One can also expect that the needs will tend to continue increasing because of household expansion and the government's policies, such as tax measures to promote ownership of lands or dwellings and personal tax reduction [4]. These very factors in turn result in the country's incapacity to generate sufficient electricity in response to the citizens' future needs since the household electricity consumption in Thailand continues rising every year.

Hence, the present study intended to develop a forecasting model for the household electricity consumption through time series models; it was conducted in the hope that the model could serve as guideline for electricity generation expansion planning to fulfill the increasing needs.

\section{BACKGROUND AND RELATED WORKS}

\section{A. Time Series}

Time series data [5], [6] can be defined as data of any items recorded at a point of time. Simply put, time series data are the data of any matter which changes over time; recorded subdata will be collected at a certain point of time, so the data are sorted or displayed in chronological order. Generally, time series data can be found in a broad array of data, for example stock market data [7], [8], electrocardiogram data [9], reservoir water level data [10] and temperature changes data [11].

\section{B. Min-Max Normalization}

Normalization [12] can be referred to a means to minimize redundancy and normalize the attributed values to a smaller range. Since the large scale data can affect processing, 
optimization of the data size is essential. There are various types of normalization, yet increasingly recognized among them is Min-Max Normalization, which was applied in this study. The so-called normalization is the process of normalizing the data to $[0,1]$ range. Normalization is illustrated in (1) below.

$\mathrm{V}^{\prime}=\frac{\mathrm{V}-\min _{\mathrm{A}}}{\max _{\mathrm{A}}-\min _{\mathrm{A}}}\left(\right.$ new_max $\left._{\mathrm{A}}-\mathrm{new}_{-} \min _{\mathrm{A}}\right)+$ new $\_$min $_{\mathrm{A}}$

where $V$ is an original attribute value.

$V^{\prime}$ is a new attribute value.

$\min _{A}, \max _{A}$ are the highest and lowest original value of attribute $\mathrm{A}$.

$n e w_{-} \min _{A}, n e w_{-} \max _{A}$ are the highest and lowest new value of attribute $A$.

\section{Stationary}

Stationary time series data have a constant mean and a variance over time. Therefore, time series data which are used to construct the model must first be tested or checked for their stationarity. In addition, the non-stationary time series data must be converted into the stationary time series data [13] by the determination of differences.

\section{Autoregressive Integrated Moving Average Model}

The Box-Jenkins approach to time series analysis [14] is a statistical methodology for identifying a forecasting model based on the relations of data in the past to build a model for explaining data behavior and forecasting the future behavior. In general, it is highly effective in forecasting the short-term time series. The model for time series analysis using the BoxJenkins approach is termed as ARIMA [15], [16], which stands for Autoregressive Integrated Moving Average. The so-called model hypothesizes that the present observation is a linear function of an observation and a past random error.

The model is displayed in (2) below.

$$
y_{t}=\delta+\emptyset_{1 y_{t-1}}+\ldots+\emptyset_{p y_{t-p}}+\epsilon_{t}-\theta_{1} \epsilon_{t-1}-\ldots-\theta_{q} \varepsilon_{t-q}
$$

where $y_{t}$ is an observation of times series at time t.

$\delta$ is a constant of a model.

$\epsilon_{t}$ is a random error at time $t$; it is hypothesized that when $\epsilon_{t}$ is an independent random variable with mean 0 , the variance is said to be homogeneous.

$\emptyset_{i}(i=1, \ldots, p)$ and $\theta_{j}(j=1, \ldots, q)$ are parameter of a model while $p$ and $q$ represent integer numbers which indicate the order of the model.

\section{E. Generalized Autoregressive Conditionally Heteroskedastic}

Engle [17] stated that most time series data have variances and are unstable. In solving that, Engle used this very model to forecast variances; specifically, conditional forecasting was used because it provided more precise predictions than unconditional forecasting did. Subsequently, he conducted a further study in which conditional variances were characterized by ARIMA [18] as displayed below.

$$
\epsilon_{t}=V_{t} \sqrt{\sigma_{t}^{2}}
$$

The variance is denoted as:

$$
\begin{gathered}
V_{t}=\sigma_{v}{ }^{2}=1 \\
\sigma_{t}^{2}=\alpha_{0}+\sum_{i=1}^{q} \alpha_{i} \varepsilon_{t-I}^{2}+\sum_{i=1}^{p} \beta_{i} \sigma_{t-I}^{2}
\end{gathered}
$$

Equation (5) illustrates the GARCH $(p, q)$ model [19]. As can be noticed, the conditional variances are constructed by Autoregressive (AR) and Moving Average (MA) features. In this equation, $\mathrm{p}$ denotes the order of GARCH Term $\left(\sigma^{2}{ }_{t-i}\right)$ while q denotes that of ARCH Term $\left(\varepsilon^{2}{ }_{t-i}\right)$.

\section{F. Triple Exponential Smoothing Method}

Triple exponential smoothing [20], also referred to as Winter's method, is a forecasting model for time series data containing trends and seasonal influences. This model is suitable for a short- to mid-term prediction. Thus, in terms of periodic attributes, daily, weekly or monthly data should be used because the use of such data will help uncover and identify seasonal components or influences. Triple exponential smoothing focuses on imbalanced data and intends to implement the smoothing on three parameters as follows:

$\alpha($ alpha $)$ is a smoothing constant between data and forecast values has a value between 0 and 1 .

$\gamma$ (gamma) is a smoothing constant between actual trends and estimated trend values has a value between 0 and 1 .

$\delta($ delta $)$ is a smoothing constant between actual seasonality and estimated seasonal values has a value between 0 and 1 .

Winters Triple Exponential Smoothing features two methods, namely Multiplicative Seasonal Model and Additive Seasonal Method. In this study, the latter method was employed with the following forecast equation denoted as below:

$$
F_{t+m}=L_{t}+b_{t}+S_{t-s+m}
$$

\section{G. Related Works}

Benvenuto et al. [21] investigated the application of the ARIMA model on the dataset of COVID-2019 epidemiology; it aimed to develop a model for forecasting the feasible evolution of this epidemic. The researcher collected data daily about the outbreak of this disease from January $20^{\text {th }}$ 2020 to February $10^{\text {th }} 2020$ in Johns Hopkins University's website and used this data to construct the ARIMA model to forecast the trends or tendency of COVID-2019 epidemiology. The results show that the ARIMA $(1,0,4)$ model was the best model for forecasting the outbreak of this disease while the ARIMA $(1,0,3)$ model was regarded as the optimal one for assessing the incidence of COVID-2019.

Jamil's research [22] on hydroelectricity consumption forecast for Pakistan using ARIMA modeling and supplydemand analysis for the year 2030 aimed to examine the hydroelectricity forecast in Pakistan. The study collected data starting 53 years back and used the ARIMA model to forecast hydroelectricity generation capacity for the year 2030. The results demonstrated that the forecasting value of hydroelectricity generation increased by 23.4 percent, accounting for 1.65 percent in each year.

Ji et al. [23] conducted a study on carbon future prices forecasting based on the ARIMA-CNN-LSTM model. The 
researcher used the ARIMA model to collect linear data of carbon prices, the CNN model to capture the data. In doing so, the dataset from April $7^{\text {th }}, 2008$ to May 6 ${ }^{\text {th }}, 2019$ was used; this dataset contained 573 weekly observations. The study revealed that the ARIMA-CNN-LSTM model could provide a more precise forecast than the standard model with respect to the root mean square and mean absolute percentage error (MAPE).

Mata et al. [24] carried out a study on forecasting coking coal prices by means of ARIMA models and neural networks. The study was to assess or investigate the efficiency in coking coal prices of traditional time series models, the ROBUST model, the ARIMA model, general regression neural networks (GRNNS), and multilayer feed-forward neural networks (MLFNs). Two cases of analysis were conducted: 1) full-time series analysis and 2) transgenic time series analysis. It was discovered that with the application of the full-time series for forecasting, the GRNN model could provide a more accurate forecast than the MLFN and ARIMA models, traditional time series models, and the ROBUST model. Conversely, as for the transgenic time series, the ARIMA model was the most accurate method.

Wang et al. [25] conducted a study on forecasting U.S. shale gas monthly production by using a metabolic nonlinear grey model and hybrid ARIMA model; forecasting on U.S. shale gas production would allow a better understanding of the market. The results indicated that the MNGM-ARIMA approach could provide a reliable forecast with 2.396 percent of errors. Thus, it can be stated that this approach could accurately forecast shale gas production and other types of fuel products.

Qiao et al. [26] carried out a study on VIX forecasting based on the GARCH-type model with observable dynamic jumps "A new perspective". This research aimed to accurately forecast India's dependence on foreign oil. Two techniques, linear Auto-Regressive Integrated Moving Average (ARIMA) and nonlinear Back Propagation (BP) were applied to develop better forecasting techniques. The forecasting has three steps: (i) integrating the metabolic idea with a nonlinear grey model to develop NMGM, (ii) integrating the proposed NMGM with ARIMA to develop NMGM-ARIMA, and (iii) develop NMGM-BP based on integrating the proposed NMGM and BP. To improve the forecasting accuracy, this work forecasted the India's dependence on foreign oil. Two models, NMGM-ARIMA and NMGM-BP were used to evaluate India's dependence on foreign oil from 1995 to 2017 and forecast the data for 2018 to 2030 . The mean relative error of the proposed forecasting models is approximately $1.5 \%$, which can produce reliable forecasting results. The forecasting results show that in 2025 India's dependence on foreign oil will be as high as $90 \%$, which challenges India's oil security and the world oil market.

Qi et al. [27] carried out a study on SPI-based drought simulation and prediction using ARMA-GARCH model. The monthly precipitation data from 1965 to 2015 from five stations in Shandong province, northern China, were used in this research to calculate the Standard Sedimentation Index (SPI) with a 9 month time scale (SPI- 9). The GARCH model was adopted to eliminate the heteroscedasticity (ARCH effect) in the residuals of ARMA (Autoregressive and Moving Average) model, and the two models were combined into a composite model called ARMA-GARCH model. The SPI-9 drought index was simulated by two models, namely ARMA and ARMA-GARCH, which results showed that the performance of model ARMA-GARCH is better than the ARMA model.

Finally, Wang et al. [28] conducted a study on the prediction of early stabilization time of electrolytic capacitor based on ARIMA-Bi_LSTM hybrid model. Hybrid models using the ARIMA model, Bi-directional long short-term memory (Bi_LSTM), and Bayesian optimization (BO) have been proposed for accuracy in predicting early performance changes concerning electrolytic capacitors. First, predicting the linear part of the time series by using ARIMA model. Then, predicting the frame for the nonlinear part with Bi_LSTM technique, the optimal experimental model was acquired through BO. Finally, the prediction result will be derived from the combination of the linear and nonlinear parts. By comparing with five models, the test results showed that the hybrid model has better prediction performance.

\section{METHOD}

The purpose of the present study was to develop a forecasting model and to compare time series forecasting using ARIMA, GARCH and Winters Triple Exponential Smoothing models for household electricity consumption datasets. In this very study, $\mathrm{R}$ language and its available package were employed to construct the suitable forecasting model. The conceptual framework is provided in Fig. 2 below.

The procedures exhibited in Fig. 2 are explained as follows:

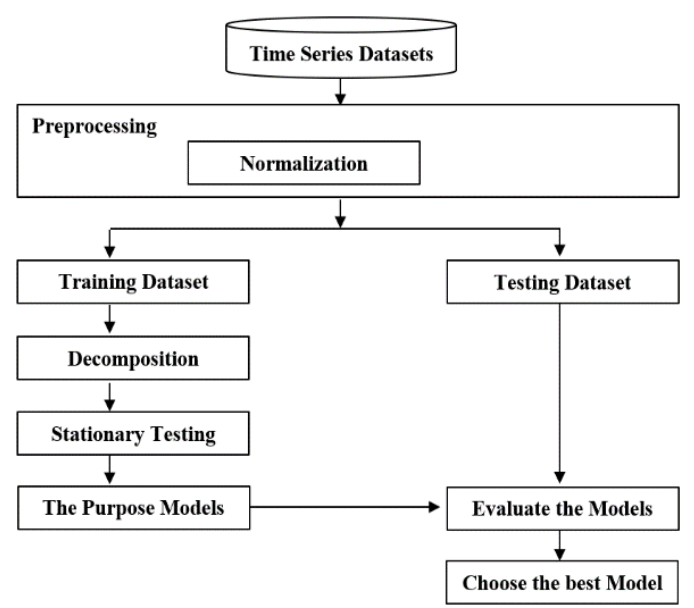

Fig. 2. The conceptual of framework.

\section{A. Data Preprocessing}

This preprocessing step involved preparing the data prior to the implementation of time series data; particularly, the data were normalized to $[0,1]$ range through Min-Max Normalization;

\section{B. Dataset Splitting}

This step was concerned with splitting or dividing monthly time series data into two sets. The first dataset, referred to as a training dataset, was used to develop the forecasting model, while the other as in a testing dataset was used to test the efficiency and errors of the forecasting model. These datasets were also used to generate time series data using Package tseries. 


\section{Decomposition}

The decomposing stage shows components of time series data; simply speaking, the time series data are decomposed into the following trends: the trend, the seasonal, the cyclical and the irregular components. The decomposition enables easier time series analysis using the decompose() function. The decomposition outcomes of datasets of household electricity consumption below and over 150 units are shown in Fig. 3 and 4 respectively.

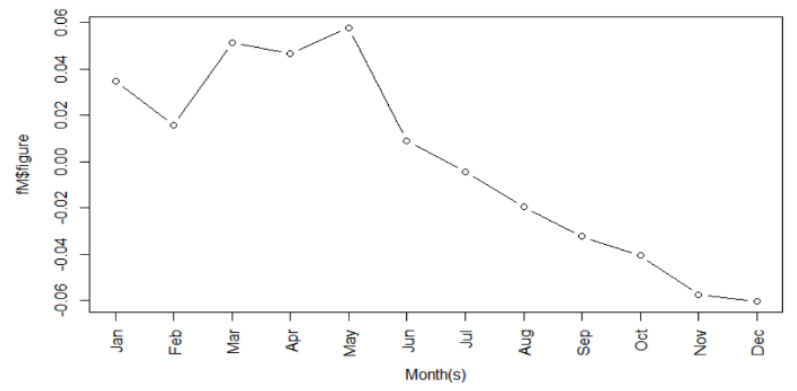

(a) Seasonal component.

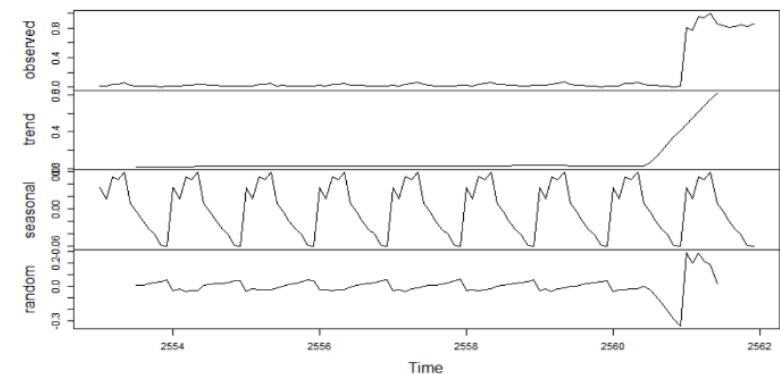

(b)Time series graph.

Fig. 3. Decomposition of time series components of household electricity consumption below 150 units.

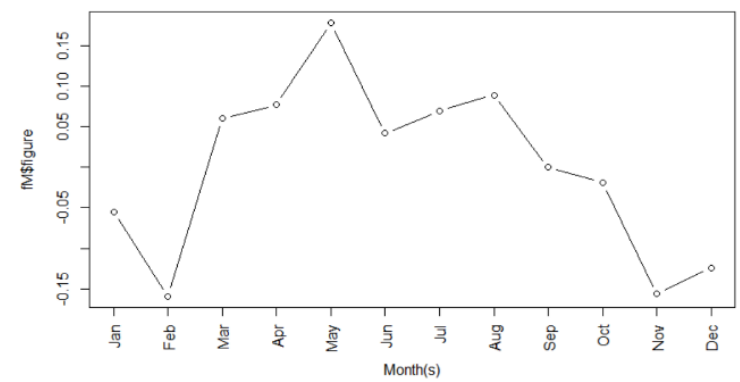

(a) Seasonal component.

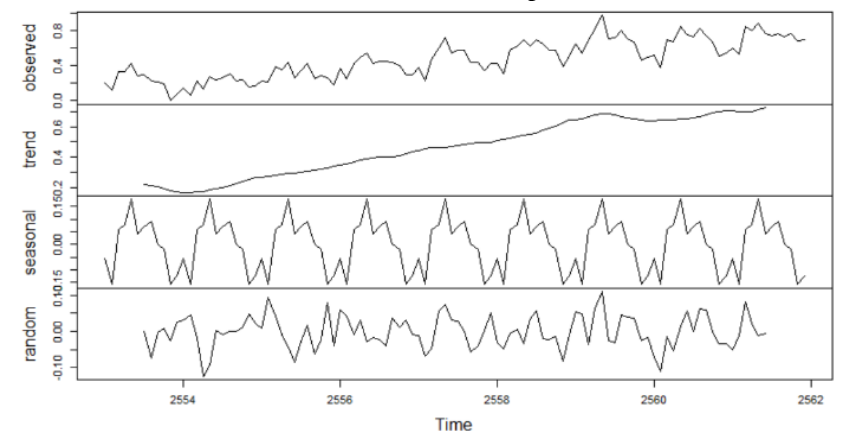

(b)Time series graph.

Fig. 4. Decomposition of time series components of household electricity consumption over 150 units.

\section{Stationary Testing}

This step involved testing data stationarity based on the ACF (Autocorrelation Function) and PACF (Partial Autocorrelation Function) graphs, performed with the $a c f()$ and $\operatorname{pacf}($ ) functions, respectively.
From Fig. 5 and Fig. 6, both datasets were nonstationary, therefore the first difference must be determined using the $\operatorname{diff}($ ) function.

After taking the first difference, both datasets were stationary as shown in Fig. 7 and Fig. 8.

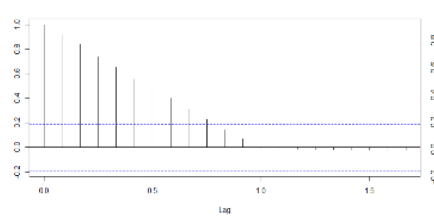

(a) ACF graph

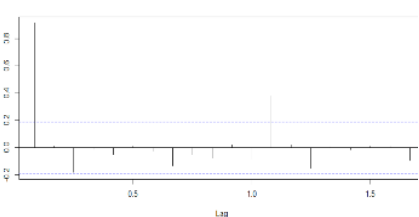

(b) PACF graph
Fig. 5. ACF and PACF graphs of monthly household electricity consumption below 150 units.

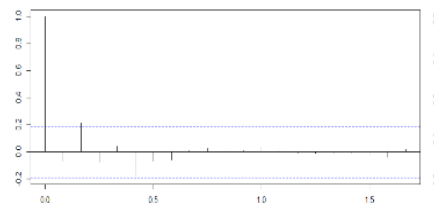

(a) ACF graph

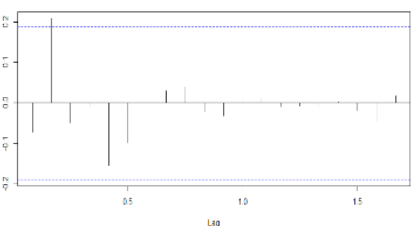

(b) PACF graph
Fig. 6. ACF and PACF graphs of monthly household electricity consumption over 150 units.

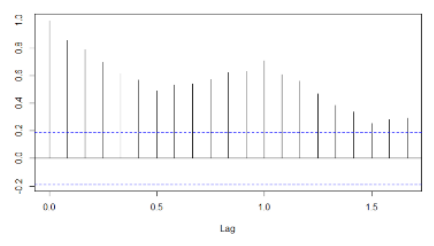

(a) ACF graph

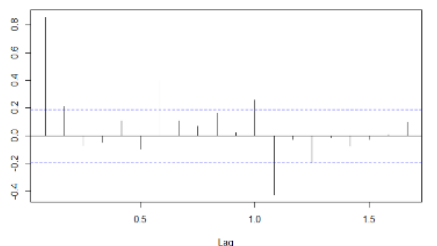

(b) PACF graph
Fig. 7. ACF and PACF graphs of monthly household electricity consumption below 150 units after first-differencing.

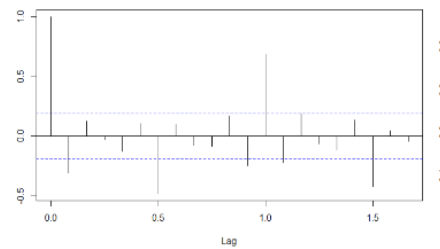

(a) $\mathrm{ACF}$ graph

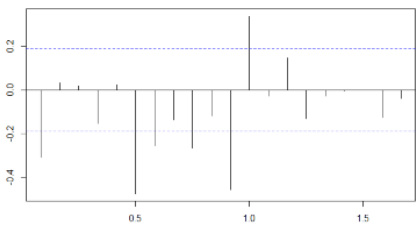

(b) PACF graph
Fig. 8. ACF and PACF graphs of monthly household electricity consumption over 150 units after first-differencing.

\section{E. Developing the Forecasting Model}

This step involved the creation of the forecasting model with three models, namely ARIMA, GARCH and Triple exponential smoothing. For ARIMA and GARCH models, the selection of the suitable forecasting models was based on an AIC value (Akaike Information Criterion); more specifically, the forecasting models with the lowest value would be selected.

The construction of the ARIMA model started with defining the order of $p, d$ and $q$ for an $\operatorname{ARIMA}(p, d, q)$ model or defining that of $p, d, q, P, D$ and $Q$ for seasonal data using an ARIMA $(p, d, q)(P, D, Q)_{m}$ model. The order of $p, d$ and $q$ was based on ACF and PACF graphs. As aforementioned, the forecasting model with the lowest value of AIC would be chosen.

The creation of the GARCH model was performed by selecting the optimal parameters $p, q$. These two parameters were defined as the input for the GARCH model using the ugarchspec() and ugarchfit() functions from the rugarch 
library. In addition, the future electricity consumption was forecasted through the ugarchforecast() function. Subsequently, the model with the lowest value of AIC would be considered as the optimal forecasting model.

Triple exponential smoothing can be performed using the $h w()$ function.

\section{F. Testing the Model Efficiency}

In this step, all forecasting models chosen through three methods in the prior step were used to provide a future forecast using the testing dataset. For a future forecast, the maximum duration of the ahead forecast was eleven months.

\section{G. Selecting the Optimal Model}

In this final step, the optimal models for each dataset would be chosen; by doing so, the forecast errors of each model were compared. The root mean squared error (RMSE) was used as a criterion; specifically, the forecasting model whose RMSE value was the lowest would be regarded as the suitable one.

\section{EXPERIMENT EVALUATION}

\section{A. Time Series Datasets}

The data used in this study were time series data of household electricity consumption. The selection of the suitable forecasting models was based on household electricity consumption data derived from Provincial Electricity Authority, Suratthani province, collected from nine districts, namely Punpin, Phanom, Khian Sa, Khiri Rat Nikhom, Tachang, Chaiya, Thachana, Ban Ta Khun and Vibhavadi. They were the monthly data from January, 2010 to November, 2019. Specifically, the datasets employed in this study were those of monthly electricity consumption fewer and higher than 150 units. Apart from that, the data were divided into two parts; in particular, the first part from January 2010 to December 2018 was applied for training, while the other between January 2019 and November 2019 was used for testing.

\section{B. Measurement of Efficiency of Forecasting Models}

The statistics used for assessment of forecasting values and tests of forecasting accuracy RMSE [29], [30] as shown in (7) below.

$$
R M S E=\sqrt{\sum \frac{(\text { prediction }- \text { actual })^{2}}{n}}
$$

\section{Results and Analysis}

The forecasting models as in ARIMA, GARCH and triple exponential smoothing were applied to provide 11-month ahead forecasts of household electricity consumption below and above 150 units. The forecasts from each model are provided in Table I, the findings are as follows:

1) Forecasts of household electricity consumption below 150 Units

In constructing the forecasting model with ARIMA, the optimal forecasting model was $\operatorname{ARIMA}(2,1,0)$ with an AIC value of -232.13 .

It could most accurately forecast one month ahead, followed by three and six months ahead, with an RMSE value of $0.0183,0.0735$ and 0.0878 respectively.

TABLE I: FORECASTS IN DIFFERENT DURATIONS OF ARIMA, GARCH AND WINTERS TRIPLE EXPONENTIAL SMOOTHING

\begin{tabular}{|c|c|c|c|c|c|c|}
\hline \multirow{2}{*}{$\begin{array}{c}\text { Dataset } \\
\text { Model }\end{array}$} & \multicolumn{3}{|c|}{ Below 150 Units } & \multicolumn{3}{|c|}{ Over 150 Units } \\
\hline & Pattern & Forecast & RMSE & Pattern & Forecast & RMSE \\
\hline \multirow[t]{3}{*}{ ARIMA } & $\operatorname{ARIMA}(2,1,0)$ & 1 & 0.0183 & $\operatorname{ARIMA}(0,0,3)(2,1,0)_{12}$ & 1 & 0.0086 \\
\hline & $(A I C=-232.13)$ & 3 & 0.0735 & $(A I C=-223.96)$ & 3 & 0.0189 \\
\hline & & 6 & 0.0878 & & 6 & 0.0659 \\
\hline \multirow[t]{3}{*}{ GARCH } & $\operatorname{GARCH}(2,0)$ & 1 & $\underline{0.0016}$ & $\operatorname{GARCH}(1,1)$ & 1 & 0.0029 \\
\hline & $(A I C=-470.15)$ & 3 & 0.0887 & $(A I C=-165.53)$ & 3 & 0.1158 \\
\hline & & 6 & 0.1281 & & 6 & 0.1953 \\
\hline \multirow{3}{*}{\multicolumn{2}{|c|}{$\begin{array}{c}\text { Triple Exponential } \\
\text { Smoothing }\end{array}$}} & 1 & 0.3798 & & 1 & 0.0515 \\
\hline & & 3 & 0.3721 & & 3 & 0.0433 \\
\hline & & 6 & 0.4084 & & 6 & 0.0405 \\
\hline
\end{tabular}
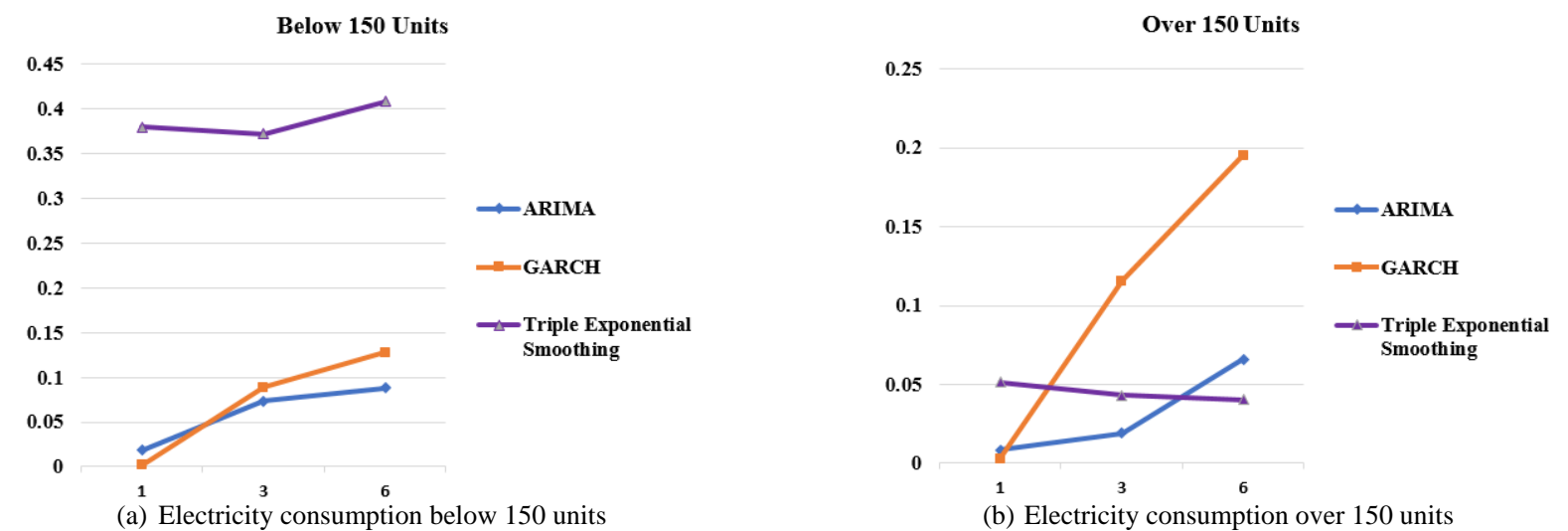

Fig. 9. The RMSE value of three forecasting models.

As for creating the forecasting model with GARCH, it was found that the suitable model was $\operatorname{GARCH}(2,0)$ with an AIC value of -470.15 ; it provided the most accurate one-month ahead forecast, followed by three and six months, with an
RMSE value of $0.0016,0.0887$ and 0.1281 respectively.

In developing the forecasting model with triple exponential smoothing, the optimal forecasting model was the one with an AIC value of 84.78834 ; it could best forecast one month 
ahead, followed by three and six months, with an RMSE value of $0.3798,0.3721$ and 0.4084 respectively.

2) Forecasts of household electricity consumption over 150 units

Based on the development of the forecasting model using ARIMA, the results showed that the most effective model was $\operatorname{ARIMA}(0,0,3)(2,1,0)_{12}$ with an AIC value of -223.96 ; it could predict one month ahead most accurately, followed by three and six months, with an RMSE value of 0.0086, 0.0189 and 0.0659 respectively.

In relation to the construction of the forecasting model based on GARCH, the most suitable model was $\operatorname{GARCH}(1,1)$ with an AIC value of -165.53 ; this model had the capacity to provide a one-month ahead forecast more accurately, followed by three and six months, with an RMSE value of $0.0029,0.1158$ and 0.1953 respectively.

The creation of the forecasting model applying triple exponential smoothing indicated that the most appropriate model was the one with an AIC value of -77.25; unlike any other models, it was able to provide most accurate six-month ahead predictions with an RMSE value of 0.0405 , followed by three and one month with that of 0.0433 and 0.0515 respectively.

\section{Selecting the Optimal Forecasting Model}

The data in Table I are displayed on the graphs in Fig. 9. As exemplified in Fig. 9(a), the GARCH models were most effective in forecasting one month ahead the household electricity consumption below 150 units, followed by ARIMA and triple exponential smoothing. On the contrary, in forecasting three and six months ahead, ARIMA could perform the predictions more accurately than GARCH and triple exponential smoothing.

In accordance with Fig. 9(b), the GARCH models could provide the most accurate one-month ahead forecasts of the household electricity consumption higher than 150 units, followed by ARIMA and triple exponential smoothing respectively. Conversely, in respect of the three-month ahead prediction, the ARIMA model was the most reliable model, whereas the GARCH models were the least. Triple exponential smoothing, among all models, could give the most accurate six-month ahead predictions.

\section{E. Forecasting Results of Household Electricity Consumption}

Based on the selection of the optimal forecasting model, the study discovered that the GARCH models were compatible with datasets of the household electricity consumption below and above 150 units and could provide a short-term ahead forecast of the electricity consumption, specifically a one-month ahead forecast.

\section{CONCLUSION}

The present study aimed to develop a model for forecasting the household electricity consumption and compare the time series forecasting models, namely ARIMA, GARCH and Triple exponential smoothing (Winter). In selecting the suitable model and pinpointing the optimal forecasting duration, the lowest values of AIC and RMSE were taken into account. Concerning the household electricity consumption below 150 units, the results revealed that the ideal forecasting period for ARIMA and GARCH was one month; in consideration of the lowest value of RMSE, GARCH was the most suitable forecasting model. As for triple exponential smoothing, the optimal forecasting duration was three months. In terms of the household electricity consumption above 150 units, the most proper period for ARIMA and GARCH was one month; based on the lowest value of RMSE, GARCH was the most applicable. Triple exponential smoothing's optimal duration was six months. Overall, it can be noticed that GARCH was compatible with both datasets and could provide the most accurate one-month ahead forecasts.

\section{CONFLICT OF INTEREST}

The authors declare no conflict of interest.

\section{AUTHOR CONTRIBUTIONS}

The works of each author in this work are as follows:

Patcharakorn Sokannit conducting research and experimenting with time series forecasting. She also prepares the material for publication.

Pasapitch Chujai is consultant and gives advice about techniques, methods to be used in the process of the time series forecasting.

All authors have approved the final version.

\section{ACKNOWLEDGMENT}

This research was supported by the Electrical Technology Education Department, Faculty of Industrial Education and Technology, King Mongkut's University of Technology Thonburi, Thailand.

\section{REFERENCES}

[1] Energy Generating Authority of Thailand, Proportion of Fuel Consumption for Electricity Generation in 2019. [Online]. Available: https://www.egat.co.th/index.php?option=com_content\&view=article \&id=2455\&Itemid $=116$

[2] Ministry of Energy, "Energy statistics of Thailand 2018," Energy Policy and Planning Office, p. 23, 2018.

[3] National Statistical Office. (2018). Energy consumption by economic sector B.E. $2010 \quad-\quad 2019 . \quad$ [Online]. Available: http://statbbi.nso.go.th/staticreport/page/sector/th/13.aspx

[4] Office of The Permanent Secretary, News of Ministry of Finance, vol. 054, p. 1, 2019.

[5] P. Sathianwiriyakhun,T. Janyakikit, and C. A. Ratanamahatana, "Fast and accurate template averaging for time series classification," International Conference on Knowledge and Smart Technology (KST), IEEE, pp. 49-54, 2016

[6] S. N. Huda, "Predicting Indonesian democracy index in Yogyakarta province as time series data using exponential smoothing," in Proc. EBIMCS '19 Conf., August 2019, no. 25, pp. 1-5.

[7] T. Bollerslev and H. O. Mikkelsen, "Modeling and pricing long memory in stock market volatility," Journal of Econometrics, vol. 73, pp. 151-184, July 1996.

[8] X. Yu and Y. Zhan, "Stability analysis of chinese stock market based on garch model," in Proc. EBIMCS '19 Conf., August 2019, no. 6, pp. 1-6.

[9] H. Sivaraks and C. A. Ratanamahatana, "Robust and accurate anomaly detection in ECG artifacts using time series motif discovery," Computational and Mathematical Methods in Medicine, p. 20, 2015.

[10] W. C. Wang, K. W. Chau, C. T. Cheng, and L. Qiu, "A comparison of performance of several artificial intelligence methods for forecasting monthly discharge time series," Journal of Hydrology, vol. 374, no. 34, pp. 294-306, August 2009.

[11] M. Ghil, and R. Vautard, "Interdecadal oscillations and the warming trend in global temperature time series," Nature, vol. 350, pp. 324-327, August 2009.

[12] C. Phipathananunth and S. Jalearnrat, "Socio- economic household data analysis using the clustering and association technique for data mining," Information and Communication Technology Program 
Faculty of Science and Technology, University of the Thai Chamber of Commerce, 2013.

[13] L. Artprom, "Volatility estimation and forecasting of the value on long term equity fund by using GARCH model," Master of Economics Independent Study, Business Economics Program, Faculty of Management Sciences, Khon Kaen University, 2012.

[14] G. Jenkins et al., Time Series Analysis Forecasting and Control, San Francisco: Holden-day, 1976.

[15] P. T. Yamak, L. Yujian, and K. Gadosey, "A comparison between ARIMA, LSTM, and GRU for time series forecasting," in Proc. ACAI the 2019 2nd International Conf., December 2019, pp. 49-55.

[16] X. Cai, Y. Liu, and Y. Xiao, "Bridge displacement prediction method based on ARIMA model," in Proc. AIIPCC '19 Conf., December 2019, no. 11 , pp. 1-6.

[17] R. F. Engle, "Autoregressive conditional heteroscedasticity with estimates of the variance of United Kingdom inflation," Econometrica, vol. 50, pp. 987-1008, July 1982.

[18] T. Bollerslev, "Generalized autoregressive conditional heteroskedasticity," Econometrics, vol. 31, pp. 307-327, 1986

[19] M. Marchese, I. Kyriakou, M. Tamvakis, and F. D. Iorio, "Forecasting crude oil and refined products volatilities and correlations: New evidence from fractionally integrated multivariate GARCH models," Energy Economics, May 2020.

[20] C. Theeraviriya, "A comparison of the forecasting method for electric energy demand in Nakhonphanom province," Master of Engineering Program, Nakhon Phanom University, 2017.

[21] D. Benvenuto, M. Giovanetti, L. Vassallo, S. Angeletti, and M. Ciccozzi, "Application of the ARIMA model on the COVID2019 epidemic dataset," Unit of Medical Statistics and Molecular Epidemiology, University Campus Bio-Medico of Rome, Italy, 2020.

[22] R. Jamil, "Hydroelectricity consumption forecast for Pakistan using ARIMA modeling and Supply-demand analysis for the year 2030," Department of Building Engineering, College of Architecture \& Planning, Imam Abdulrahman Bin Faisal University, 2020.

[23] L. Ji, Y. Zou, K. He, and B. Zhu, "Carbon futures price forecasting based with ARIMA-CNN-LSTM model," School of Business, Hunan University of Science and Technology, 2018.

[24] M. Marta, P. R. Fernández, A. Krzemien, K. Wodarski, and G. F. Valverde, "Forecasting coking coal prices by means of ARIMA models and neural networks, considering the transgenic time series theory," Resources Policy, vol. 61, pp. 283-292, 2019.

[25] Q. Wang, S. Li, R. Li, and M. Ma, "Forecasting U.S. shale gas monthly production using a hybrid ARIMA and metabolic nonlinear grey model," Energy, vol. 160, pp. 378-387, October 2018.

[26] G. Qiao, J. Yanga, and W. Li, "VIX forecasting based on GARCH-type model with observable dynamic jumps: A new perspective," The North American Journal of Economics and Finance, vol. 53, no. 101186, July 2020.

[27] L. Qi, Z. Guanlan, S. Ali, W. Xiaopeng, W. Guodong, P. Zhenkuan, and Z. Jiahua, "SPI-based drought simulation and prediction using
ARMA-GARCH model," Applied Mathematics and Computation, vol. 355, pp. 96-107, August 2019.

[28] Z. Wang, J. Qu, X. Fang, H. Li, T. Zhong, and H. Ren, " Prediction of early stabilization time of electrolytic capacitor based on ARIMABi_LSTM hybrid model," Neurocomputing, vol. 403, pp. 63-79, August 2020.

[29] T. Chai and R. R. Draxler, "Root mean square error (RMSE) or mean absolute error (MAE)? - Arguments against avoiding RMSE in the literature," Geoscientific Model Development, vol. 7, no. 3, pp. 1247 1250,2014

[30] J. W. Koo, S. W. Wong, G. Selvachandran, H. V. Long, and L. H. Son, "Prediction of Air Pollution Index in Kuala Lumpur using fuzzy time series and statistical models," Air Quality, Atmosphere \& Health, vol 13, pp. 77-88, 2020

Copyright $\odot 2021$ by the authors. This is an open access article distributed under the Creative Commons Attribution License which permits unrestricted use, distribution, and reproduction in any medium, provided the original work is properly cited (CC BY 4.0).

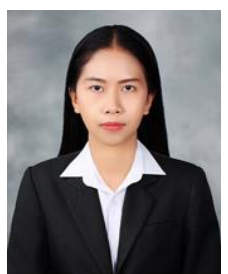

Patcharakorn Sokannit is a teacher at Phunphinpitthayakom School, Suratthani province. She received her bachelor's degree in computer engineering at the Electrical Technology Education Department, Faculty of Industrial Education and Technology, King Mongkut's University of Technology Thonburi, Thailand in 2017. Currently, she is studying at master's degree level in computer engineering at the Electrical Technology Education Department, FIET, KMUTT. Her current research includes time series and machine learning and data processing.

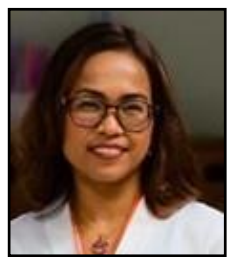

Pasapitch Chujai is a lecturer at the Electrical Technology Education Department, Faculty of Industrial Education and Technology, King Mongkut's University of Technology Thonburi, Thailand. She received her bachelor degree in computer science from Ramkhamhaeng University, Thailand, in 2000, her master degree in computer and information technology from King Mongkut's University of Technology Thonburi, Thailand, in 2004 and her doctoral degree in Computer Engineering, Suranaree University of Technology, Thailand, in 2015. Her current research includes ontology, recommendation system, time series, machine learning, and imbalanced data classification. 